\title{
A Simplified Equivalent Circuit Model of MEMS Electrostatic Actuator
}

\author{
Pradeep Chawda \\ Senior Member \\ IEEE \\ Bangalore (INDIA)
}

\begin{abstract}
Modeling a MEMS (Micro Electro-Mechanical Systems) electrostatic actuator in electrical domain is important for system simulation of the actuator along with its associated electronics. For instance, an integrated MEMS resonator used in a serial I/O PLL design modeled in electrical domain enables to optimize the system with the rest of the electronics. In this work, we have developed a simplified equivalent circuit model for MEMS electrostatic actuator and simulated it using Natspice, a U.C. Berkeley SPICE3f5-based in-house circuit simulator. The equations governing the actuator are implemented using coupled RL and RLC circuit, defined in SPICE and Verilog-A. Natspice simulation results are presented and compared with Matlab results which show very high correlation. A system consisting of an array of MEMS devices can be quickly simulated using this simplified model.
\end{abstract}

\section{General Terms}

MEMS, Modelling, Simulation, MATLAB, SPICE, Verilog-A

\section{Keywords}

Microactuator, Equivalent Circuit, Large Signal, Small Signal

\section{INTRODUCTION}

In recent times MEMS-based actuators are integrated in microelectronics, as against standalone transducers in the past, to build a complex system [1-3]. A system-level simulation of MEMS actuators along with peripheral electronics is required in order to design and optimize the performance of the system. Modeling and simulation of MEMS actuators usually involves use of multi-domain analysis tools [4] such as MATLAB [5], ANSYS [6, 7] and SUGAR [8]. In order to design and optimize a system with MEMS actuators and associated electronics in an IC design environment, the designers manually pass on the MEMS actuator performance parameters for circuit simulation, which is time consuming and error prone. This paper presents implementation of an equivalent circuit model of MEMS actuator in SPICE/Verilog-A to describe the transient behavior of the electrostatic actuator. The model takes in to account the effect of source resistance and can deal with both small and large amplitude input signals. During pull-in, the model restricts the gap to predefined minimum value so that it can be simulated successfully with a circuit simulator. The model can be simulated using any circuit simulator capable of simulating SPICE and Verilog-A. There are several equivalent circuit models which have been developed [9-19], however this paper presents a simplified model which is accurate as well as fast and can used for system level simulations[20-21].

\section{EQUIVALENT CIRCUIT MODEL FOR ELECTROSTATIC ACTUATOR}

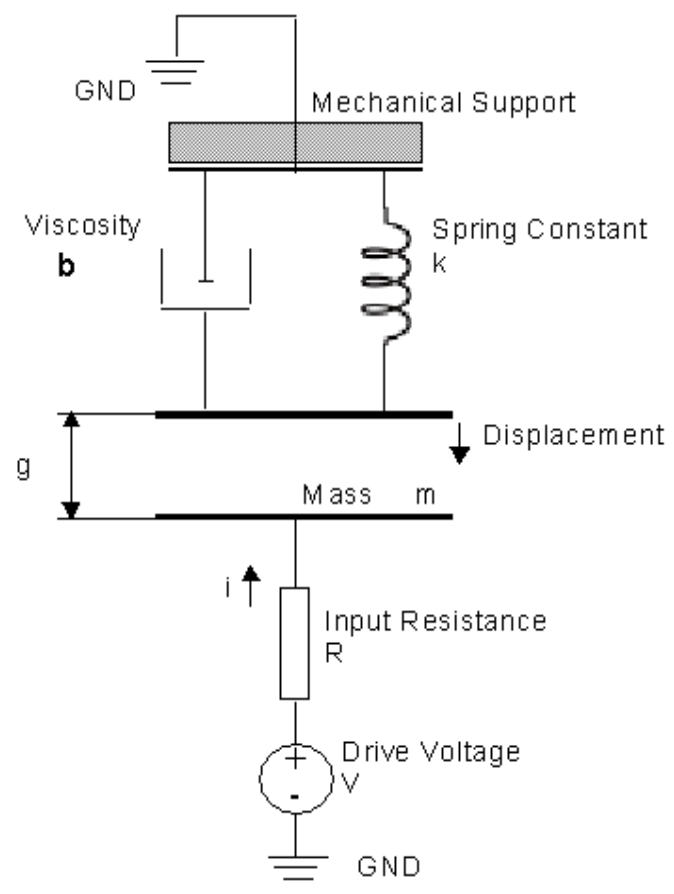

Figure 1: Electrostatic actuator model

Figure 1 shows schematic of a parallel-plate electrostatic actuator. Equations (1) to (5) describe the plate's motion $[22,23]$

$$
\text { Electrostatics }\left\{\begin{array}{l}
F=\frac{1}{2} \varepsilon \frac{A}{(g)^{2}} V_{i n}{ }^{2} \\
Q=\varepsilon \frac{A}{g} V_{i n}
\end{array}\right.
$$

Equation of motion $\quad \ddot{g}=\left(F-b \cdot \dot{g}-k \cdot\left(g-g_{0}\right)\right) \frac{1}{m}$

Kirchoff's Laws $\left\{\begin{aligned} V & =R \cdot i+V_{\text {in }} \\ i & =\frac{d}{d t}\left(C \cdot V_{\text {in }}\right)\end{aligned}\right.$

The list of parameters used in equation (1) to (5) (and values that were used for simulations) are shown in Table 1 . These 
equations are usually solved in MATLAB/Simulink [2]. In order to simulate a system with the electrostatic actuator in the electrical domain we have developed an equivalent circuit that models the switching behavior of the actuator using electrical circuit elements and yet governed by the set of equations describing the plate's motion.

Table 1: List of parameters and its values

\begin{tabular}{|c|c|c|}
\hline Symbol & Parameter & $\begin{array}{l}\text { Value used in } \\
\text { Simulation }\end{array}$ \\
\hline$\varepsilon_{0}$ & $\begin{array}{l}\text { Dielectric Constant of free } \\
\text { space }\end{array}$ & $8.85 \times 10^{-12}$ \\
\hline$\varepsilon$ & $\begin{array}{l}\text { Dielectric Constant of } \\
\text { medium used in } \\
\text { simulation }\end{array}$ & $1.67 \times 10^{-11}$ \\
\hline $\mathrm{E}$ & $\begin{array}{l}\text { Young's Modulus } \\
\text { (silicon) }\end{array}$ & $160 \mathrm{Gpa}$ \\
\hline$\rho$ & Density (silicon) & $2.33 \times 10^{3} \mathrm{~kg} / \mathrm{m}^{3}$ \\
\hline G & Gap between the plates & -- \\
\hline $\mathrm{F}$ & Electrostatic Force & -- \\
\hline $\mathrm{Q}$ & Induced Electric Charge & -- \\
\hline $\bar{A}$ & Plate Area & $(1.67 \mathrm{um})^{2}$ \\
\hline K & Spring Constant & $5.13 \times 10^{4} \mathrm{~N} / \mathrm{m}$ \\
\hline $\mathrm{M}$ & Plate mass & $4.14 \mathrm{e}-7 \mathrm{~kg}$ \\
\hline $\mathrm{B}$ & Viscosity & $7.18 \times 10^{-4} \mathrm{~N}-\mathrm{s} / \mathrm{m}$ \\
\hline $\mathrm{g}_{0}$ & Electrostatic Initial Gap & $1.04 \times 10^{-5} \mathrm{~m}$ \\
\hline $\mathrm{C}$ & Capacitance $(\varepsilon \mathrm{A} / \mathrm{g})$ & - \\
\hline $\mathrm{V}_{\text {in }}$ & $\begin{array}{c}\text { Drive Voltage to Fixed } \\
\text { Plate }\end{array}$ & -- \\
\hline-- & $\begin{array}{l}\text { Drive Voltage to movable } \\
\text { plate }\end{array}$ & GND 0v \\
\hline $\mathrm{R}$ & Input Resistance & $50 \Omega$ to $2 \mathrm{M} \Omega$ \\
\hline $\mathrm{V}$ & Input Voltage & -- \\
\hline
\end{tabular}

\subsection{Developing equivalent circuit for electrostatic actuator}

By combining (2), (4) and (5) we can write (6) as:

$$
\dot{Q}=I=\frac{1}{R}\left(V-\frac{Q g}{\varepsilon A}\right) .
$$

And by combining (1) and (3) we can write (7) as:

$$
\frac{Q^{2}}{2 \varepsilon A}+b \dot{g}+m \ddot{g}+k\left(g-g_{0}\right)=0
$$

By solving (6) we can get the value of charge, Q (with initial condition $\mathrm{g}=\mathrm{g}_{0}$ ) and by solving (7) we can get the value of gap, g. These two equations are coupled differential equations and can be modeled as RL and RLC circuits, respectively. The complete equivalent circuit model is shown in Figure 2.
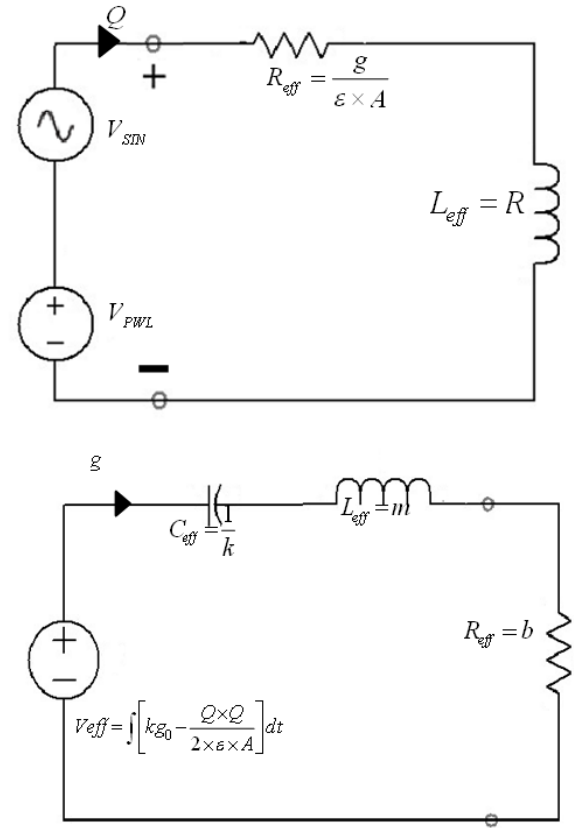

Figure 2: Equivalent circuit model of electrostatic actuator

2.2 Deriving effective values of circuit elements

For a RL circuit, the Kirchoffs Voltage Law (KVL) gives:

$$
\dot{I}=\frac{1}{L}(V-I R) .
$$

By comparing (6) with (8) we can say that the current (I) in the RL circuit is numerically equal to charge $(\mathrm{Q})$ of equation (6) if the effective inductance and the effective resistance of the RL circuit are given by:

$$
R_{e f f}=\frac{g}{\varepsilon A}, L_{e f f}=R \text {. }
$$

Therefore, we can solve equation 6 by using a RL circuit. Similarly, the KVL in RLC circuit gives:

$$
L \dot{I}+R I+\frac{1}{C} \int I d t=V .
$$

Assuming zero initial conditions and differentiating (10) gives:

$$
L \ddot{I}+R \dot{I}+\frac{I}{C}=\dot{V} .
$$

Comparing (7) and (11) we can say that the current (I) in the RLC circuit is numerically equal to gap (g) of (7) if the effective resistance, inductance, capacitance and input voltage of RLC circuit are given by:

$$
\begin{aligned}
& R_{\text {eff }}=b, L_{\text {eff }}=m, C_{\text {eff }}=1 / k \\
& V_{\text {eff }}=\int\left(k g_{0}-\frac{Q^{2}}{2 \varepsilon A}\right) d t
\end{aligned}
$$

Since the initial gap is $\mathrm{g}_{0}$, then the initial current flowing through the inductor in RLC circuit must be numerically equal to $\mathrm{g}_{0}$. 


\subsection{Simulating pull-in effect}

The model developed so far will simulate properly if the input voltage is less than the pull-in voltage [2] given by:

$$
V_{P I}=\sqrt{\frac{8 k g_{0}{ }^{3}}{27 \varepsilon A}} .
$$

If the input voltage is more than pull-in voltage, then the gap will become zero and the simulator will not be able to successfully simulate the model and will give nonconvergence errors. In order to properly simulate the pull-in effect, we should restrict the gap (g) to a certain minimum value, say $g_{\min }$. This can be included in the model by using an arbitrary voltage source along with a ternary behavioral operator:

$$
g_{\text {eff }}=g>g_{\text {min }} ? g: g_{\text {min }}
$$

The $\mathrm{g}_{\text {eff }}$ is used in the RL circuit to take in to account the pullin effect in the model.

\subsection{Calculating various output parameters}

The voltage across the electrostatic actuator plates is calculated from (2) and given by:

$$
V_{i n}=\frac{Q g}{\varepsilon A},
$$

The velocity of the movable plate of electrostatic actuator is calculated by differentiating the gap (g) using an inductor of $1 \mathrm{H}$ and a current source of value g connected across inductor. Voltage developed across the inductor is the velocity of the moveable plate of electrostatic actuator.

\section{SIMULATION RESULTS AND DISCUSSION}

In order to simulate the actuator model, we have used an inhouse circuit simulator, Natspice. The netlist has been created for the model using SPICE circuit elements and a Verilog-A module to perform integration (using idt() construct of Verilog-A ) to calculate the effective voltage that is applied to the RLC circuit.

The Verilog-A model used in the SPICE simulation is instantiated as $\mathrm{P}$ device (P1) with .model card specifying the Verilog-A module name q_calc. A subcircuit with name cscmut_va is created to model the coupled RL and RLC circuit. Apart from the RL and RLC circuits it has the ' $V$ ' and ' $\mathrm{B}$ ' devices to easily access the output parameters such as voltage across the plates, pressure, gap and velocity. The subcircuit is instantiated and current source is applied to the model. The reltol is set to 1e-4 for better accuracy. A transient analysis is performed and simulation results are captured. In order to validate the results of presented model, we have created a Matlab/simulink model and compared the simulation results. The complete SPICE netlist along with Verilog-A model definition is provided below for reference. With little syntax changes it can be simulated with any other SPICE simulator capable of simulating Verilog-A.

//A Verilog-A model to calculate idt $(\mathrm{k} * \mathrm{~g} 0-\mathrm{Q} 2 / 2 \mathrm{eA})$

include "constants.vams"

include "disciplines.vams"

module q_calc(q_2, q_value); inout q_2, q_value;

electrical q_2, q_value,q_tmp;

parameter real $\mathrm{A}=1.67 \mathrm{e}-6 ; / / \mathrm{Area}(\mathrm{m} 2)$

parameter real e $=1.47 \mathrm{e}-11 ; / /$ Dielectric $\operatorname{constant}(\mathrm{F} / \mathrm{m})$

parameter real $\mathrm{g} 0=1.04 \mathrm{e}-5 ; / / \operatorname{gap}(\mathrm{m})$

parameter real $\mathrm{k}=5.13 \mathrm{e} 4 ; \quad$ // Spring constant $(\mathrm{N} / \mathrm{m})$

parameter real $\mathrm{m}=4.14 \mathrm{e}-7$; // Plate mass $(\mathrm{Kg})$

analog begin

$\mathrm{V}\left(\mathrm{q} \_\right.$value $)<+\mathrm{idt}\left(\mathrm{k} * \mathrm{~g} 0-\left(\mathrm{V}\left(\mathrm{q} \_2\right) * \mathrm{~V}\left(\mathrm{q} \_2\right)\right) /(2 * \mathrm{e} * \mathrm{~A})\right)$;

end

endmodule

*Netlist for current source CMUT cell

.subckt cscmut_va Iin_in Fin_in vout gdot g Q Pressure PARAMS:

$+\mathrm{e}=1.47 \mathrm{e}-11$

$+\mathrm{A}=1.67 \mathrm{e}-6$

$+\mathrm{g} 0=1.036 \mathrm{e}-5$

$+\mathrm{b}=7.1829999 \mathrm{e}-4$

$+\mathrm{m}=4.14351 \mathrm{e}-7$

$+\mathrm{k}=5.13 \mathrm{e} 4$

$+\mathrm{R}=1 \mathrm{e} 12$

*** END OF .SUBCKT STATEMENT

*** MEASUREMENTS $* * *$

***Voltage across the plates

Bvol vout $0 \mathrm{~V}=\mathrm{V}(\mathrm{g}) * \mathrm{~V}(\mathrm{Q}) * 41000 /(\{\mathrm{e}\} *\{\mathrm{~A}\})$

***Charge across the plates

BQ Q 0 V=I(VB5)

** Verilog-A model instantiation

P1 Q Q_value Pmod

.model Pmod q_calc

****Gap across the plate $\mathrm{g}$

BG_val g 0 V=I(VB1)

****Velocity gdot

Bgdot gdot $0 \mathrm{I}=\mathrm{V}(\mathrm{g})$

Lgdot gdot 01

***Pressure generated by the plates

Bpressure pressure $0 \mathrm{~V}=\mathrm{V}($ gdot $)<-1 \mathrm{e} 4$ ? 0 : $\mathrm{V}($ gdot $) * 415$

*** EQUIVALENT CIRCUIT ***

$* * * *$ RL Circuit $* * * *$

Bq1 input $0 \mathrm{~V}=\mathrm{I}(\mathrm{VI})$

BRes Input Q_rl I=V(Input, Q_rl $) *\left(\{\mathrm{e}\}^{*}\{\mathrm{~A}\}^{*}\{\mathrm{R}\}\right) / \mathrm{V}(\mathrm{g})$

L2 Q_rl tt 1

VB5 tt 00 
VI 0 Iin_in 0

$$
\text { **** END RL Circuit **** }
$$$$
\text { **** RLC Circuit **** }
$$

B1 G_src $0 \quad \mathrm{~V}=\mathrm{V}$ (Q_value)

R3 G_src G_rl $\{b\}$

L1 G_rl G_lc $\{\mathrm{m}\} \mathrm{IC}=\{\mathrm{g} 0\}$

C2 G_lc ttt $\{1 / \mathrm{k}\}$

VB1 ttt 00

$$
\begin{aligned}
& * * * * \text { END RLC CIRCUIT **** } \\
& \text {.ends } \\
& * * * \text { End of subckt } \\
& * * * \text { TESTBENCH } * * * * \\
& * * * \text { Instantiating subckt }
\end{aligned}
$$

Xcmut Iin_in 0 vout gdot g Q Pressure cscmut_va

\section{***SIN excitation}

IVsin Iin_in $0 \operatorname{SIN}(00.5 \mathrm{u} 5.6 \mathrm{e} 48.9 \mathrm{~m})$

$* * *$ Bias excitation

IVtri Iin_in 0 pwl(0 $04.5 \mathrm{~m} 1.023454987372858 \mathrm{e}-06$ 8.9m 0)

***Setting up Transient Analysis

.tran 100n 12.5m 0 100n UIC

***Simulator options for high accuracy

.options reltol $=1 \mathrm{e}-4$

.end

\section{*** END of SPICE NETLIST}

Figure 3 shows the overlaid plots. The input current is shown in fig 3(a). The source resistance used in the model for the current source is $1 \mathrm{e} 12$ as can be seen from the netlist. The input current has two components, one is PWL for biasing and another is SIN for excitation. As soon as biasing current is applied the charge across the plates builds up and when the biasing current becomes zero, the charge remains constants as can be seen from Figure 3(b). However the MEMS actuator starts to oscillate as can be seen from Figure 3(c). The developed charge across the actuator plates is shown in Figure 3(b). We can see a very good match of Natspice simulation result with Matlab model. The normalized gap is shown in Figure 3(c). The gap is normalized with respect to pull-in voltage. Figure 4(a) shows the voltage across the actuator plates. Velocity of the moveable plate is shown in Figure 4(b). As can be seen from the overlaid plots, Natspice results are in very good agreement with Matlab results. This validates the model.

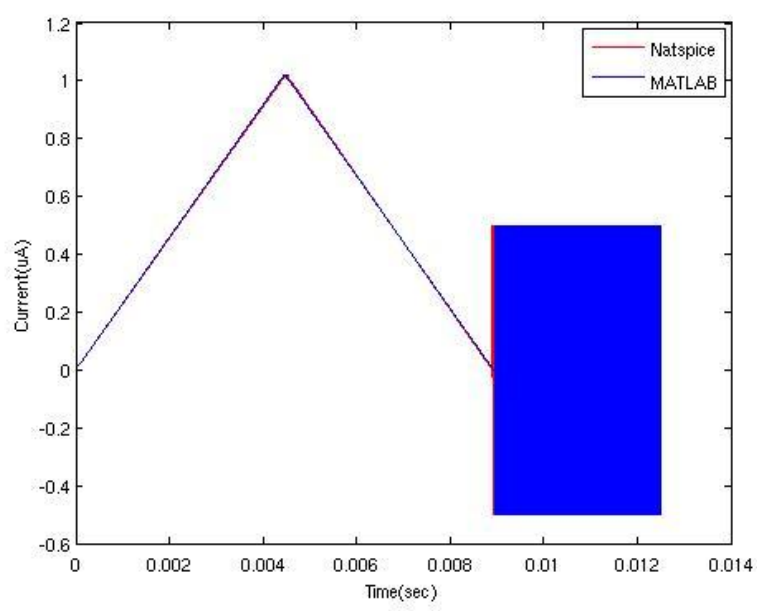

(a)

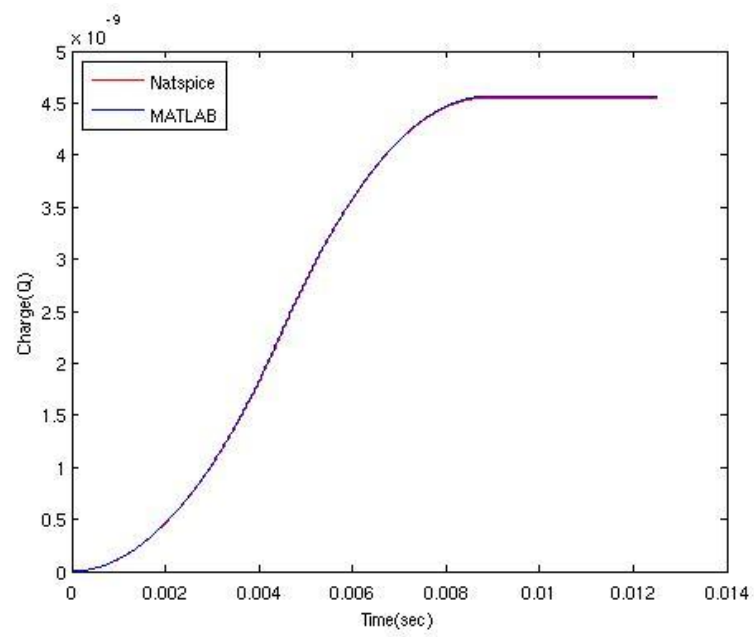

(b)

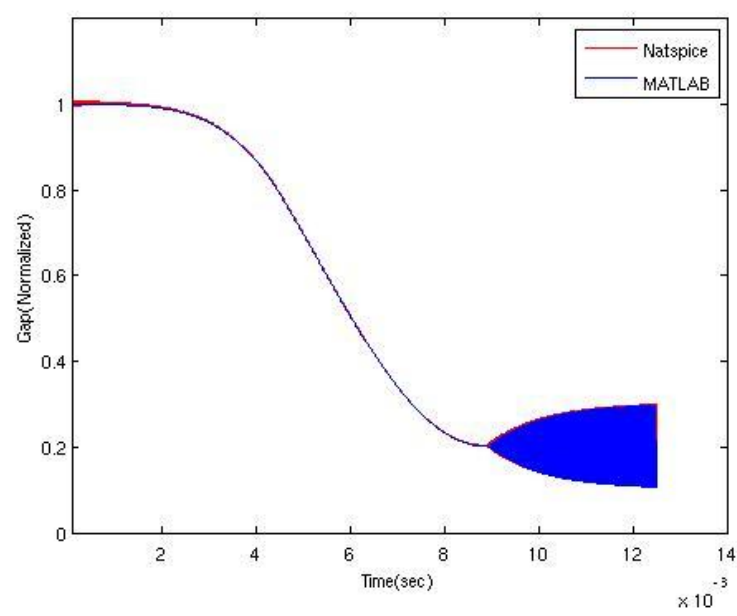

(c)

Figure 3: Comparison of Natspice simulation results with Matlab results, (a) Current (b) Charge and (c) Normalized Gap 


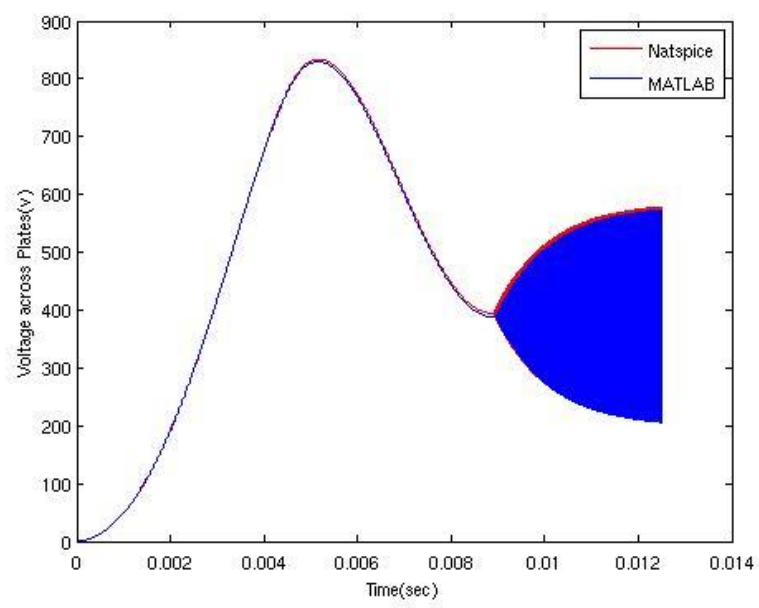

(a)

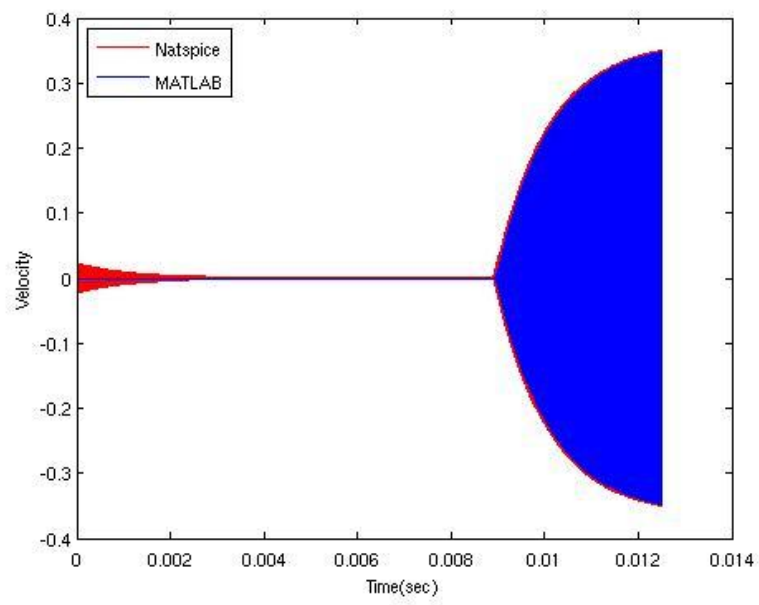

(b)

Figure 4: Comparison of Natspice simulation results with Matlab results, (a) Voltage across actuator plates (b) Velocity of movable plate.

An arbitrary voltage source is used to limit the value of gap (g) as given in (14). Simulation plots were normalized with respect to pull-in voltage. The input voltage applied can be a sine wave and a triangular wave to observe small and large amplitude behavior of electrostatic actuator. For large signal input we applied a triangular wave as shown in Figure 3. The developed charge is shown in Figure 5(a). Figure 5(b) shows that when the input voltage is less then pull-in voltage the moveable plate comes back to its initial position otherwise plate remains at $\mathrm{g}_{\min }$. Figure $5(\mathrm{c})$ shows the pull-in phenomenon which happens at $2 / 3 \mathrm{~g}_{0}$ with $\mathrm{g}_{\min }=3$ (normalized). Therefore gap does not become zero. Figure 6(a) shows the pull-in and release process in gap vs charge plot. Figure 6(b) shows the actuator gap vs time plot for source resistance of $2 \mathrm{M} \mathrm{ohm}$ and $\mathrm{g}_{\min }=3$ along with applied voltage. In Figure 6(c) the actuator plate velocity and normalized input voltage vs. time is plotted. The pull-in and release can be seen by positive peak and negative valley in the velocity plot.

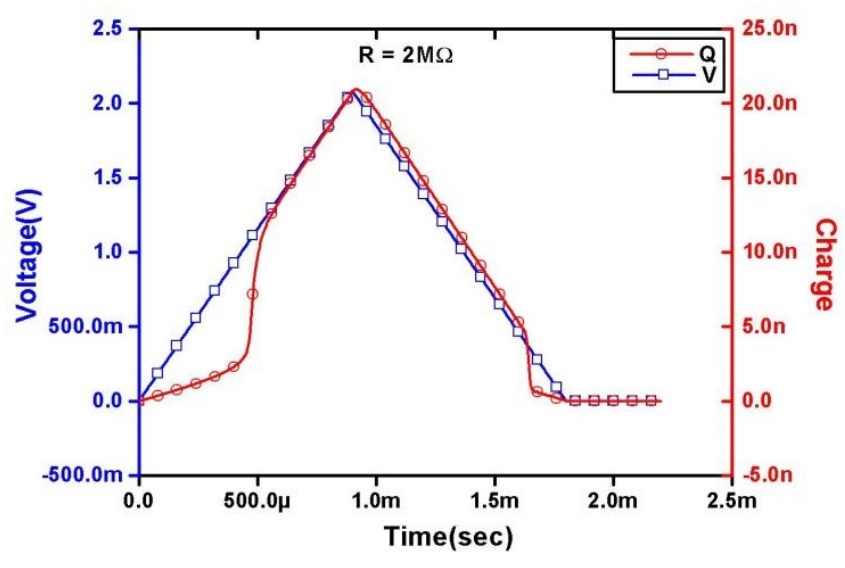

(a)

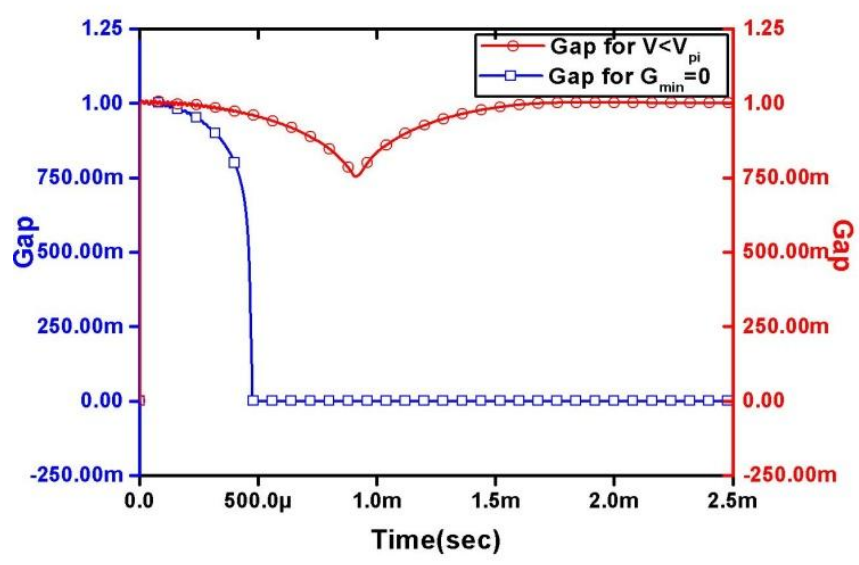

(b)

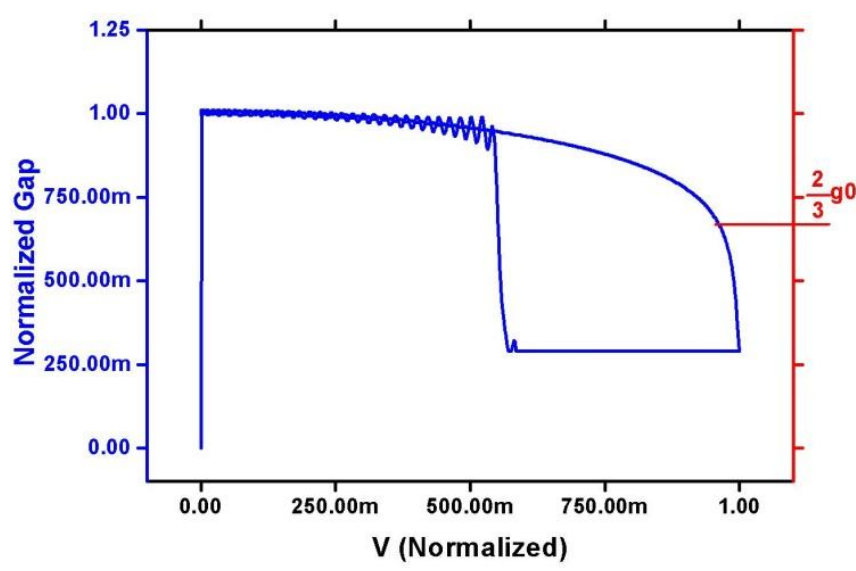

(c)

Figure 5: Natspice simulation results, (a) applied voltage and developed charge vs. time plot for source resistance of $2 \mathrm{M} \Omega$. (b) plot of actuator gap vs. time with input voltage, less then pull-in voltage and otherwise (c) plot of actuator gap vs. normalized input voltage 


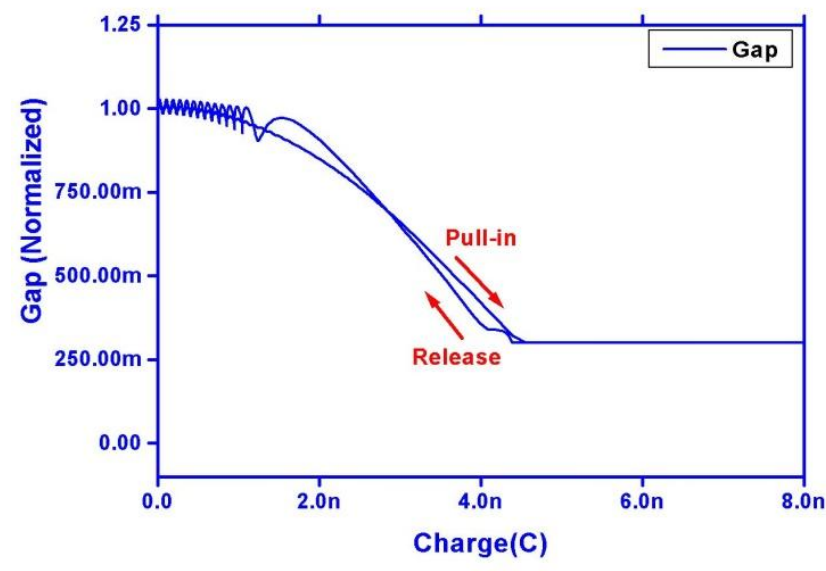

(a)

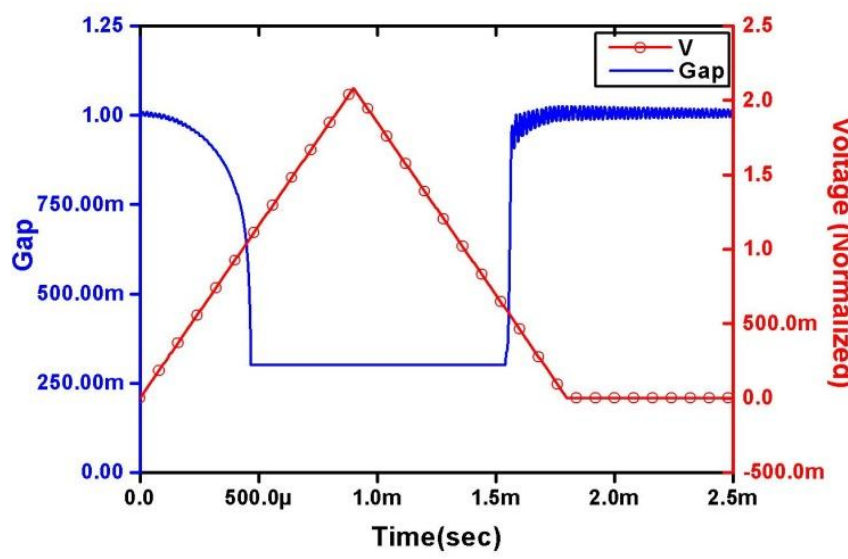

(b).

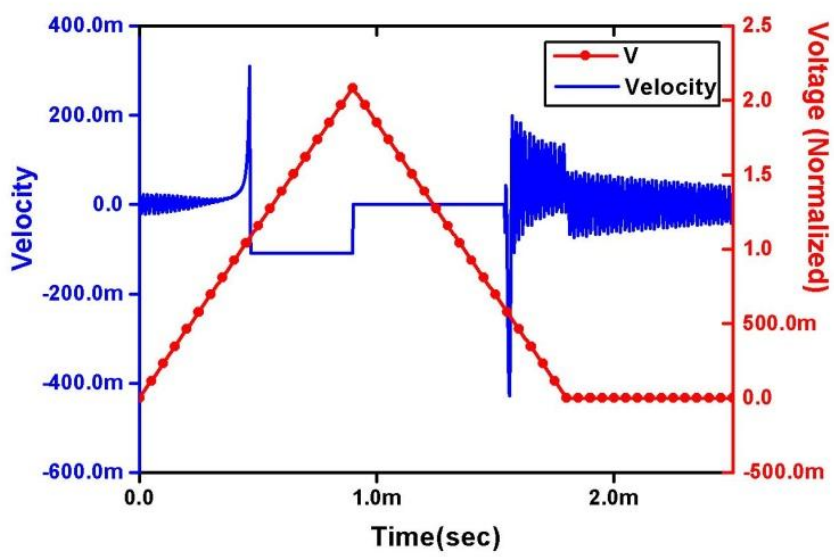

(c)

Figure 6: Simulation results, (a) plot of actuator gap vs. charge across the actuator plates (b) applied voltage and actuator gap vs. time plot with source resistance of $2 \mathrm{M} \Omega$ and $g_{\min }=3$ (c) plot of actuator plate velocity and normalized input voltage vs. time
The effect of source resistance can be observed in the gap vs. time plot in Figure 7(a) and voltage vs. time plot Figure 7(b) respectively. Figure 7(c) shows the response of small amplitude input voltage to the model. The input is a triangular

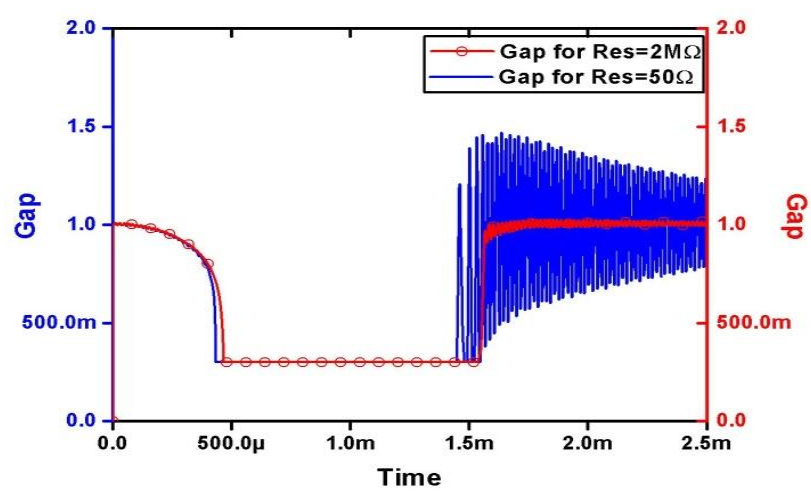

(a)

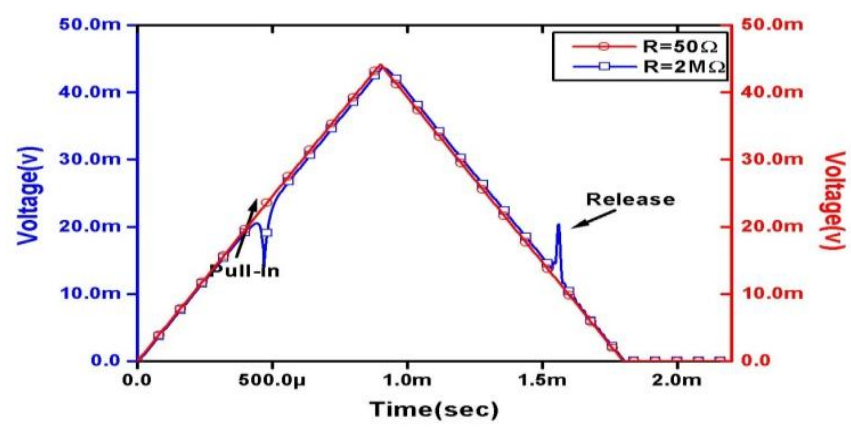

(b)

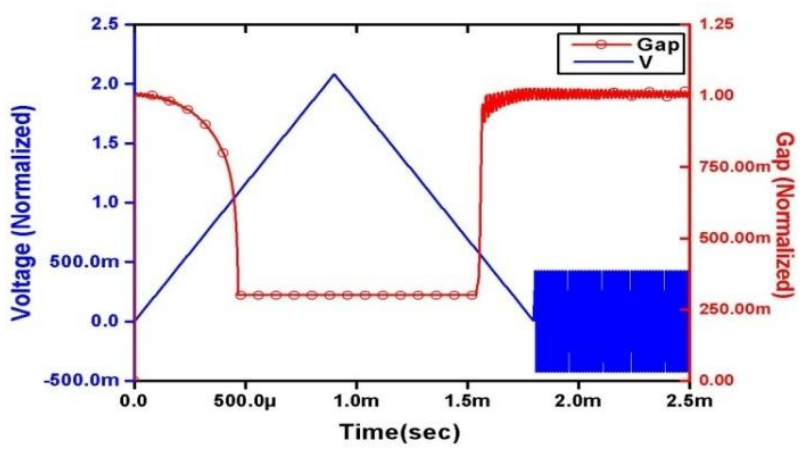

(c)

Figure 7: Simulation results, (a) gap vs. time for source resistance of 50 and $2 \mathrm{M} \Omega$ (b) plate voltage vs. time for source resistance of 50 and $2 \mathrm{M} \Omega$ (c) plot of small amplitude signal superimposed on large amplitude triangular wave as input voltage and actuator gap vs. time

Voltage pulse with superimposed sine wave and the resultant actuator oscillation (gap between the actuator plates) is shown in the figure.

\section{CONCLUSION}

We presented a coupled RL and RLC equivalent circuit model for a MEMS electrostatic actuator and proved its correctness 
by derivation. Natspice simulation results of the actuator model implemented in SPICE and Verilog-A were discussed. The response of the actuator to small and large amplitude input signal and the effect of source resistance were demonstrated.

\section{REFERENCES}

[1] Schröpfer, Gerold, et al. "Advanced process emulation and circuit simulation for co-design of MEMS and CMOS devices." Proceedings Symposium on Design, Test, Integration and Packaging of MEMS/MOEMS (DTIP). 2005.

[2] Cheng, J., Zhe, J., Wu, X., Farmer, K. R., Modi, V., \& Frechette, L. (2002, May). Analytical and FEM simulation pull-in study on deformable electrostatic micro actuators. In Technical Proc. of the International Conf on Modeling and Simulation of Microsystems, MSM (pp. 298-301).

[3] Wang, C. K., Chen, C. S., \& Wen, K. A. (2011, May). A monolithic CMOS MEMS accelerometer with chopper correlated double sampling readout circuit. In Circuits and Systems (ISCAS), 2011 IEEE international Symposium on (pp. 2023-2026). IEEE.

[4] Senturia, S. D. (1998). CAD challenges for microsensors, microactuators, and microsystems. Proceedings of the IEEE, 86(8), 1611-1626.

[5] Cheng, J., Zhe, J., Wu, X., Farmer, K. R., Modi, V., \& Frechette, L. (2002, May). Analytical and FEM simulation pull-in study on deformable electrostatic micro actuators. In Technical Proc. of the International Conf on Modeling and Simulation of Microsystems, MSM (pp. 298-301).

[6] Camon, H., Larnaudie, F., Rivoirard, F., \& Jammes, B. (1999, May). Analytical simulation of a 1D single crystal silicon electrostatic micromirror. In International Conference on Modeling and Simulation of Microsystems (pp. 628-631).

[7] Certon, D., Teston, F., \& Patat, F. (2005). A finite difference model for cMUT devices. IEEE transactions on ultrasonics, ferroelectrics, and frequency control, 52(12), 2199-2210.

[8] Clark, J. V., Zhou, N., \& Pister, K. S. J. (1998, June). MEMS simulation using SUGAR v0. 5. In Solid-State Sensor and Actuator Workshop (pp. 191-196).

[9] Lohfink, A., Eccardt, P. C., Benecke, W., \& Meixner, H. (2003, October). Derivation of a 1D CMUT model from FEM results for linear and nonlinear equivalent circuit simulation. In Ultrasonics, 2003 IEEE Symposium on (Vol. 1, pp. 465-468). IEEE.

[10] Lohfink, A., \& Eccardt, P. C. (2005). Linear and nonlinear equivalent circuit modeling of CMUTs. IEEE transactions on ultrasonics, ferroelectrics, and frequency control, 52(12), 2163-2172.

[11] Wygant, I. O., Kupnik, M., \& Khuri-Yakub, B. T. (2008, November). Analytically calculating membrane displacement and the equivalent circuit model of a circular CMUT cell. In Ultrasonics Symposium, 2008. IUS 2008. IEEE (pp. 2111-2114). IEEE.

[12] Mita, M., \& Toshiyoshi, H. (2009). An equivalent-circuit model for MEMS electrostatic actuator using opensource software Qucs. IEICE Electronics Express, 6(5), 256-263.

[13] Sathe, A. A., Groll, E. A., \& Garimella, S. V. (2008). Analytical model for an electrostatically actuated miniature diaphragm compressor. Journal of micromechanics and microengineering, 18(3), 035010.

[14] Cicek, İ., Bozkurt, A., \& Karaman, M. (2005). Design of a front-end integrated circuit for $3 \mathrm{D}$ acoustic imaging using 2D CMUT arrays. IEEE transactions on ultrasonics, ferroelectrics, and frequency control, 52(12), 2235-2241.

[15] Konishi, T., Machida, K., Masu, K., \& Toshiyoshi, H. (2013). Multi-Physics Equivalent Circuit Model for MEMS Sensors and Actuators. ECS Transactions, 50(14), 55-61.

[16] Koymen, H., Atalar, A., Aydogdu, E., Kocabas, C., Oguz, H. K., Olcum, S., ... \& Unlugedik, A. (2012). An improved lumped element nonlinear circuit model for a circular CMUT cell. IEEE transactions on ultrasonics, ferroelectrics, and frequency control, 59(8).

[17] Wygant, I. O., Kupnik, M., \& Khuri-Yakub, B. T. (2016, September). CMUT design equations for optimizing noise figure and source pressure. In Ultrasonics Symposium (IUS), 2016 IEEE International (pp. 1-4). IEEE.

[18] Oguz, H. K., Atalar, A., \& Köymen, H. (2013). Equivalent circuit-based analysis of CMUT cell dynamics in arrays. IEEE transactions on ultrasonics, ferroelectrics, and frequency control, 60(5), 1016-1024.

[19] Oguz, H. K., Atalar, A., \& Köymen, H. (2013). Equivalent circuit-based analysis of CMUT cell dynamics in arrays. IEEE transactions on ultrasonics, ferroelectrics, and frequency control, 60(5), 1016-1024.

[20] Savoia, A. S., Caliano, G., \& Pappalardo, M. (2012). A CMUT probe for medical ultrasonography: from microfabrication to system integration. IEEE transactions on ultrasonics, ferroelectrics, and frequency control, 59(6), 1127-1138.

[21] Song, J., Jung, S., Kim, Y., Cho, K., Kim, B., Lee, S. \& Kim, D. (2012, February). Reconfigurable 2D cMUTASIC arrays for 3D ultrasound image. In SPIE Medical Imaging (pp. 83201A-83201A). International Society for Optics and Photonics.

[22] Gurun, G., Tekes, C., Zahorian, J., Xu, T., Satir, S., Karaman, M., ... \& Degertekin, F. L. (2014). Single-chip CMUT-on-CMOS front-end system for real-time volumetric IVUS and ICE imaging. IEEE transactions on ultrasonics, ferroelectrics, and frequency control, 61(2), 239-250.

[23] Senturia, S. D. (2007). Microsystem design. Springer Science \& Business Media.

[24] Hung, E. S., \& Senturia, S. D. (1999). Extending the travel range of analog-tuned electrostatic actuators. Journal of microelectromechanical systems, 8(4), $497-$ 505 .

[25] Seeger, J. I., \& Boser, B. E. (2003). Charge control of parallel-plate, electrostatic actuators and the tip-in instability. Journal of Microelectromechanical systems, 12(5), 656-671. 